\title{
Analysis of Multipactor Effect in a Partially Dielectric-Loaded Rectangular Waveguide
}

\author{
Andrés Berenguer, Ángela Coves ${ }^{\circledR}$, Member, IEEE, Francisco Mesa ${ }^{\circledR}$, Fellow, IEEE, Enrique Bronchalo ${ }^{\circledR}$, \\ and Benito Gimeno ${ }^{\circledR}$, Member, IEEE
}

\begin{abstract}
This paper presents a study of the multipactor effect in a partially dielectric-loaded rectangular waveguide. To obtain the simulations presented in this paper, a detailed analysis of the dynamics of the electron inside this waveguide has been performed, taking into account the radio frequency electromagnetic fields propagating in the waveguide and the dc electric field that appears because of the charging of the dielectric layer. This electrostatic field is obtained by computing the electric potential produced by an arbitrary charge distribution on the dielectric layer in a dielectric-loaded waveguide. The electron trajectory is then found by numerically solving the equations of motion. The results obtained show that multipactor discharges do turn off by themselves under certain circumstances when they occur in such dielectric-loaded waveguide.
\end{abstract}

Index Terms-Multipactor, secondary emission, waveguide.

\section{INTRODUCTION}

$\mathbf{M}$ ULTIPACTOR is a high-power resonant electron discharge frequently observed in the microwave and millimeter-wave subsystems operating under vacuum conditions [1] present in a wide range of different scenarios, such as passive components of satellite communication payloads, traveling-wave, tubes or particle accelerators. In an ultrahigh vacuum environment, the free electrons inside a microwave device are accelerated by the radio frequency (RF) electromagnetic fields, impacting against its metallic walls. If the electron impact energy is high enough, one or more secondary electrons might be released from the surface. When some resonance conditions are satisfied, the secondary electrons get synchronized with the RF fields, and the electron population inside the device grows exponentially leading to a multipactor discharge. This multipactor discharge has some negative effects that degrade the device performance: increase

Manuscript received May 9, 2018; revised October 3, 2018; accepted November 4, 2018. This work was supported in part by the Agencia Estata de Investigación and in part by the Unión Europea through the Fondo Europeo de Desarrollo Regional-Una Manera de Hacer Europa under Project TEC2016-75934-C4-2-R and Project TEC2017-84724-P. The review of this paper was arranged by Senior Editor S. J. Gitomer. (Corresponding author: Angela Coves.)

A. Berenguer, A. Coves, and E. Bronchalo are with the Department of Communication Engineering, Miguel Hernandez University of Elche, 03202 Alicante, Spain (e-mail: angela.coves@umh.es).

F. Mesa is with the Microwaves Group, Department of Applied Physics 1, Escuela Técnica Superior de Ingenieria Informatica, Universidad de Seville, 41004 Seville, Spain.

B. Gimeno is with the Department of Applied Physics and Electromagnetism, Institute of Materials Science, Universidad de Valencia, 46010 València, Spain.

Color versions of one or more of the figures in this paper are available online at http://ieeexplore.ieee.org.

Digital Object Identifier 10.1109/TPS.2018.2880652 of signal noise and reflected power, heating of the device walls, outgassing, detuning of resonant cavities, and even the partial or total destruction of the component.

Multipactor research lines are aimed to study and characterize the phenomenon to predict the conditions for its appearance [2], [3]. Some RF devices, such as filters, multiplexers, and RF satellite payloads, include dielectric materials commonly employed as resonators and supporting elements. In [4], it is presented a review of multipactor discharge on metals and dielectric windows that takes into account the surface materials, and the effects of space charge and cavity loading. The multipactor effect including the presence of dielectric materials in single-surface multipactor regime has been widely investigated in the context of particle accelerators; for instance, in ceramic RF windows [5], [6] and in aluminabased dielectric-loaded accelerating structures [7]. In contrast, very few contributions can be found about multipactor breakdown on dielectrics in the scenario of RF systems for space applications [8]-[10], and mostly under the parallelplate waveguide approximation. In [11] and [12], the effective electron model (EEM) has been successfully used for simulations of multipaction experiments in coaxial transmission lines considering the presence of external magnetic static fields, demonstrating the validity of this method in complex scenarios. The multipactor inside an empty rectangular waveguide has also been studied in [13] and [14], where the conventional resonance theory gives correct predictions for the multipactor threshold if the height of the waveguide is very small and first-order resonance multipactor dominates. When the waveguide height exceeds a certain critical value, which depends on the waveguide width, an accurate prediction of the multipactor threshold requires considering the RF fields inside the waveguide without approximations. Therefore, there is a need to accurately predict the electron discharge on devices involving partially dielectric-loaded rectangular waveguides, which are of more practical interest for satellite technology. The main aim of this investigation is to extend the results of previous works [8]-[10], where an EEM was successfully applied to study the multipactor in a parallel-plate dielectricloaded waveguide, to the analysis of multipactor effect in a partially dielectric-loaded rectangular waveguide.

In Section II, the theoretical model employed for the simulations is discussed. In Section III, the multipactor prediction results of an empty rectangular waveguide are analyzed and compared with results from the technical literature for validation purposes. Then, the susceptibility chart of a partially 


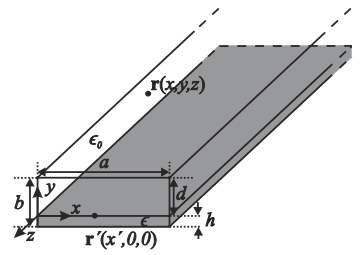

Fig. 1. Geometry and dimensions of the problem under investigation.

dielectric-loaded rectangular waveguide is obtained with the developed model, and the time evolution of a discharge in this waveguide is studied and discussed. Finally, Section IV outlines the main conclusions of this paper.

\section{THEORY}

\section{A. Computation of RF and DC Fields in a Partially Dielectric-Loaded Waveguide}

Fig. 1 shows the scheme of a partially dielectric-loaded rectangular waveguide of width $a$ and height $b$, and whose dielectric material has relative permittivity $\epsilon_{r}$. In the problem under study, the dielectric slab of thickness $h$ and width $a$ is placed over the bottom waveguide wall, being $d$ the empty waveguide height where the effective electron travels (see Fig. 1). The RF electromagnetic field is assumed to propagate along the positive direction of the $z$-axis. For the sake of simplicity, the waveguide is supposed to be infinite along the $z$ direction, and a time-harmonic dependence of the type $e^{j \omega t}$ is implicitly assumed, with $f=\omega / 2 \pi$ being the frequency and $t$ the time measured in the laboratory reference system. To analyze the multipactor evolution in this waveguide, a multipactor simulation code based on the Monte Carlo method has been developed. The software code, similar to the one described in [8] and [9], employs the single EEM [15]. This assumption avoids the consideration of space-charge effects, what is a strong simplification. The space-charge effects are often neglected in the analysis of the first stages of the multipactor discharge [13], [14], but they are doubtless important at high electron populations when the discharge is fully developed. Simulation results of some published works [16], [17] indicate an important role of space-charge in the evolution of the multipactor process to a saturation stage. In this paper, however, we are mainly interested in studying the influence of dielectric charging in the multipactor process. The inclusion of space-charge effects, although providing a more realistic description of the global process, would increase the computational burden very much, as the dc field due to dielectric charging has to be evaluated in every effective electron position. In addition, the interpretation of simulation results would become difficult, as dielectric charging and space charge can both lead to a repulsion of the freshly emitted secondary electrons back to the surface.

The effective electron at $\mathbf{r}=(x, y, z)$ can move in the air region of height $d$ of the rectangular waveguide. The electromagnetic fields $\mathbf{E}_{\mathrm{RF}}$ and $\mathbf{H}_{\mathrm{RF}}$ acting on the effective electron correspond to the modes of the partially dielectricloaded rectangular waveguide (Fig. 1), which are hybrid modes of $\mathrm{TM}^{y}$ and $\mathrm{TE}^{y}$ kinds [18]. We have restricted our study to the monomode regime, where only the fundamental mode, $\mathrm{TM}_{10}^{y}$, propagates in the waveguide. The instantaneous field vectors interacting with the effective electron are given by

$$
\begin{aligned}
\mathbf{E}_{\mathrm{RF}}(x, y, z, t) & =E_{0} \Re\left(\mathbf{e}(x, y) e^{j\left(\omega t-\beta z+\varphi_{0}\right)}\right) \\
\mathbf{H}_{\mathrm{RF}}(x, y, z, t) & =H_{0} \Re\left(\mathbf{h}(x, y) e^{j\left(\omega t-\beta z+\varphi_{0}\right)}\right)
\end{aligned}
$$

where $\varphi_{0}$ is the initial phase and $E_{0}, H_{0}$ are the constants related to the transmitted power in the waveguide. The modal fields $\mathbf{e}(x, y)$ and $\mathbf{h}(x, y)$ and the propagation constant $\beta$ of the $\mathrm{TM}_{10}^{y}$ mode can be found in [18] and [19]. These expressions can be directly extended if higher order modes must be taken into account (i.e., in waveguide discontinuities) by using the mode-matching technique.

The key to understanding the mechanism of a multipactor discharge is to study the behavior of the electrons within the waveguide, which are accelerated by the aforementioned electromagnetic fields $\mathbf{E}_{\mathrm{RF}}$ and $\mathbf{H}_{\mathrm{RF}}$. In this way, sooner or later, these fields will make an electron impact with any surface of the rectangular waveguide, which can result in the emission or absorption of secondary electrons. If the impacts occur on the dielectric surface, unlike the case of impacts on the metallic walls, the secondary electrons emitted by the dielectric give rise to positive charges at the impact positions on the dielectric surface, while the electrons absorbed in the dielectric layer will generate negative charges in it. These charges, which are located on the dielectric surface at positions $\mathbf{r}^{\prime}=\left(x^{\prime}, 0, z^{\prime}\right)$, give rise to an electrostatic field $\mathbf{E}_{\mathrm{dc}}$, which has to be added to the RF fields to obtain accurately the trajectory of the electrons inside the waveguide. In order to determine the electrostatic field, $\mathbf{E}_{\mathrm{dc}}(x, y, z)=-\nabla \phi(x, y, z)$, generated by the charges on the dielectric, the potential $\phi(x, y, z)$ inside the waveguide has to be first calculated. Using the superposition, the potential in the waveguide due to the set of charges $Q_{i}$ on the dielectric surface can be obtained by adding the individual contribution of each charge

$$
\phi(x, y, z)=\sum_{i} G\left(x-x_{i}^{\prime}, y,\left|z-z_{i}^{\prime}\right|\right) Q_{i}\left(x_{i}^{\prime}, 0, z_{i}^{\prime}\right)
$$

where $G(x, y, z)$ is the electrostatic potential due to a unit point charge, that is, Green's function for this problem.

The above-mentioned Green's function, $G(x, y, z)$, is the solution to the following Laplace's equation [20], [21]:

$$
\nabla \cdot\left[\epsilon_{r}(y) \nabla G(x, y, z)\right]=-\frac{1}{\epsilon_{0}} \delta\left(x-x^{\prime}\right) \delta(y) \delta(z)
$$

where $\epsilon_{0}$ is the free-space dielectric permittivity and the position of the unit charge is taken at $\left(x^{\prime}, 0,0\right)$ for convenience. Both the geometric characteristics and the linear nature of the problem under consideration makes that the Dirac delta functions can be expressed as [21]

$$
\begin{aligned}
\delta\left(x-x^{\prime}\right) & =\frac{2}{a} \sum_{n=1}^{\infty} \sin \left(k_{x n} x\right) \sin \left(k_{x n} x^{\prime}\right) \\
\delta(z) & =\frac{1}{2 \pi} \int_{-\infty}^{\infty} e^{-j k_{z} z} d k_{z}
\end{aligned}
$$

where $k_{x n}=n \pi / a$ and $k_{z}$ is the spectral Fourier variable along the longitudinal direction $z$. The above-mentioned expressions 
come from the fact that the eigenfunctions of the differential operator are sinusoidal functions along $x$-axis and complex exponential functions along the $z$-axis, respectively. This is equivalent to apply the discrete sine transform along the $x$-axis and the integral transform along the $z$-axis, namely,

$$
\begin{aligned}
G & =\frac{1}{\pi a} \int_{-\infty}^{\infty} d k_{z} e^{-j k_{z} z} \sum_{n=1}^{\infty} \sin \left(k_{x n} x\right) \sin \left(k_{x n} x^{\prime}\right) \widetilde{G} \\
\widetilde{G} & =\int_{-\infty}^{\infty} d z e^{j k_{z} z} \sum_{n=1}^{\infty} \sin \left(k_{x n} x\right) \sin \left(k_{x n} x^{\prime}\right) G
\end{aligned}
$$

where $G=G\left(x, x^{\prime}, y, z\right)$ and $\widetilde{G}=\widetilde{G}\left(k_{x n}, k_{z} ; y\right)$.

According to the above-mentioned considerations, (3) can be expressed as the following ordinary differential equation for the spectral Green's function $\widetilde{G}$ :

$$
\begin{aligned}
\left\{\frac{d}{d y} \epsilon_{r}(y) \frac{d}{d y}-k_{t}^{2}\right\} \widetilde{G} & =-\frac{\delta(y)}{\epsilon_{0}} \\
\widetilde{G}(y=-h) & =0 \\
\widetilde{G}(y=d) & =0
\end{aligned}
$$

where $k_{t}^{2}=k_{x n}^{2}+k_{z}^{2}$. Solving (8), the following expression for $\widetilde{G}$ is obtained in the air region $y \geq 0$ :

$$
\widetilde{G}\left(k_{x n}, k_{z} ; y\right)=\frac{\sinh \left[k_{t}(d-y)\right]}{\epsilon_{0} k_{t}\left[\epsilon_{r} \operatorname{coth}\left(k_{t} h\right)+\operatorname{coth}\left(k_{t} d\right)\right] \sinh \left(k_{t} d\right)} .
$$

Green's function in the spatial domain, $G$, is achieved by replacing (9) into (6) to give

$$
\begin{aligned}
& G\left(x, x^{\prime}, y, z\right) \\
& =\frac{2}{\epsilon_{0} \pi a} \sum_{n=1}^{\infty} \sin \left(k_{x n} x\right) \sin \left(k_{x n} x^{\prime}\right) \\
& \quad \times \int_{0}^{\infty} \frac{\sinh \left[k_{t}(d-y)\right] \cos \left(k_{z} z\right)}{k_{t}\left[\epsilon_{r} \operatorname{coth}\left(k_{t} h\right)+\operatorname{coth}\left(k_{t} d\right)\right] \sinh \left(k_{t} d\right)} d k_{z}
\end{aligned}
$$

In (10), if the point charge is placed at $z^{\prime} \neq 0, z$ must be replaced by $\left(z-z^{\prime}\right)$. Here, it is worth noting that very efficient numerical summation and integration techniques have to be employed to compute Green's function with sufficient accuracy and tolerable CPU times [22].

Once Green's function has been calculated, the $\mathbf{E}_{\mathrm{dc}}$ field is obtained by numerical differentiation of (2) by means of the central difference technique.

\section{B. Multipactor Evolution in the Partially Dielectric-Loaded Waveguide}

Once the RF and dc fields are known at any instant $t$, the electron dynamics inside the waveguide can be computed, which is governed by the Lorentz force and related to its linear momentum

$$
\mathbf{F}_{L}=q(\mathbf{E}+\mathbf{v} \times \mathbf{B})=\frac{d \mathbf{p}}{d t}
$$

where $q=-e$ is the electron charge, $\mathbf{E}$ and $\mathbf{B}=\mu_{0} \mathbf{H}$ are the total electric and magnetic fields (both RF and dc contributions) interacting with the electron, $\mu_{0}$ is the freespace magnetic permeability, and $\mathbf{v}$ is the velocity vector of the electron. The linear relativistic momentum is defined as

$$
\mathbf{p}=m_{0} \gamma \mathbf{v}
$$

where $m_{0}$ is the electron rest mass, $\gamma=1 / \sqrt{1-(v / c)^{2}}$ the Lorentz factor, $v$ is the magnitude of the velocity vector, and $c=1 / \sqrt{\mu_{0} \epsilon_{0}}$ the speed of light in vacuum. Although the relativistic correction in this equation can be discarded for the typical power ranges of most space waveguide devices, it must be considered when high velocities are reached $(v / c \geq 0.1)$, as in high-power multipactor simulations. Expanding (11), the following differential equation is obtained:

$$
-\mathbf{E}-\mathbf{v} \times \mathbf{B}=M \gamma \mathbf{a}+\frac{M}{c^{2}} \gamma^{3}(\mathbf{v} \cdot \mathbf{a}) \mathbf{v}
$$

where $\mathbf{a}$ is the acceleration vector and $M=m_{0} / e$. The differential equation to be solved becomes

$$
\ddot{\mathbf{r}}=\frac{-\dot{\mathbf{r}} \times \mathbf{B}-\mathbf{E}+\dot{\mathbf{r}} \cdot(\dot{\mathbf{r}} \cdot \mathbf{E}) / c^{2}}{M \gamma} .
$$

The electron trajectory is found by numerically solving the above-mentioned equations of motion. For that purpose, a Velocity Verlet algorithm [23] has been used, which assures sufficient accuracy and good efficiency provided the time step is small enough. Regarding this last point, in order to improve the accuracy and efficiency of the simulation, the following adaptive time step has been applied in the proximity of the waveguide walls, depending on the electron position:

$$
\Delta t=\frac{\Delta t_{0}}{1+\xi\left(\frac{x-a / 2}{a / 2}\right)^{2}+\xi\left(\frac{y-d / 2}{d / 2}\right)^{2}}
$$

where $\Delta t_{0}$ is the initial reference time step, $\xi$ is a constant value (in this case, a value of 4.0 has been chosen), and $x$ and $y$ are the coordinates of the electron position.

As mentioned above, the computed electrons trajectories may lead to an eventual impact with a surface. Each collision can result in the emission or absorption of secondary electrons. A relevant growth in the electron density can develop if the electrons hit the walls with the appropriate energy and at suitable instants. The number of electrons emitted or absorbed after each impact is determined by the value of the Secondary Electron Yield (SEY) parameter $\delta(\delta>1$ if secondary electrons are emitted, and $\delta<1$ if they are absorbed). The SEY is modeled by a modification of Vaughan's model [24] that includes the effect of reflected electrons for low impact energies of primary electrons, which has to be accounted for to obtain accurate results [25], [26] in agreement with experimental data obtained in [27], [28]. The SEY properties for surface materials can be defined by the following parameters: the primary electron impact kinetic energies which yield $\delta=1$, $W_{1}$, and $W_{2}$; the impact energy $W_{\max }$ necessary for a primary electron to yield $\delta=\delta_{\max }$, which is the maximum value of the SEY function; and the value of the primary electron impact energy $W_{0}(\delta=0)$ that limits the region of elastic collisions.

When a multipactor discharge evolves in the partially dielectric-loaded waveguide under study, the dc field distribution has to be updated after each electron impacts on the 
TABLE I

SECONDARy ElECtron EMISSiON Yield Properties of DifFERENT MATERIALS [31], [32]

\begin{tabular}{|c|c|c|c|c|c|}
\hline Material & $\mathbf{W}_{\max }(\mathbf{e V})$ & $\mathbf{W}_{\mathbf{1}}(\mathbf{e V})$ & $\mathbf{W}_{\mathbf{2}}(\mathbf{e V})$ & $\mathbf{W}_{\mathbf{0}}(\mathbf{e V})$ & $\delta_{\max }$ \\
\hline Niobium & 200.0 & 33 & 1500 & - & 1.6 \\
\hline Silver & 165.0 & 30 & 5000 & 15.99 & 2.22 \\
\hline Teflon & 271.7 & 27 & 5000 & 6.81 & 2.47 \\
\hline
\end{tabular}

dielectric surface. However, tracking the evolution of all the electrons involved in the multipactor discharge would suppose a big computational cost. Thus, we have made use of an EEM, but considering both the spread in secondary emission energy and the angle of the secondary electrons after each impact on the waveguide walls. This assumption has proved to account properly for the charging of the dielectric material, given that the discharging time for dielectrics is much higher than the typical time for a multipactor discharge. Thus, in the EEM assumed in this paper, after the effective electron impacts at time $t$ with any surface, $N_{i}(t)$ is modified according to the $\delta$ value provided by the SEY function as follows:

$$
N_{i}(t+\Delta t)=\delta N_{i}(t)
$$

where $N_{i}(t)$ represents the population of the ee inside the waveguide at the instant $t$, and $\Delta t$ is the time step used in the simulations.

The secondary electron departure kinetic energy $E_{s}$ after each electron impact is assumed to fit the following probability density function [29]:

$$
\frac{d p\left(E_{s}\right)}{d E_{s}}=C \exp \left[-\frac{\ln ^{2}\left(E_{s} / E_{m}\right)}{2 \tau^{2}}\right]
$$

where $C$ is a normalization constant, the parameter $\tau$ (typical values 0.7-0.8) determines the width of the distribution and $E_{m}$ (typical values 3-4 eV) is the energy of the maximum of the spectrum. Finally, the secondary electrons after inelastic impacts are emitted following a cosine distribution of the polar angle.

\section{NUMERICAL RESULTS AND DISCUSSION}

An in-house simulation computer-aided design (CAD) tool based on the Monte Carlo method described in Section II has been developed to analyze the multipactor effect in partially dielectric-loaded rectangular waveguides. The first problem analyzed consists of an empty rectangular waveguide previously studied in [14], whose multipactor prediction results have been used for validation purposes. The rectangular waveguide has dimensions $a=43.2 \mathrm{~cm}$ and $b=10.2 \mathrm{~cm}$, and is excited by a time-harmonic signal at $f=500 \mathrm{MHz}$. The material of the waveguide walls is niobium, whose SEY properties are given in Table I and can be expressed with the simple model proposed in [30]. In the algorithm of the simulator used in [14], for each RF power considered in the waveguide, the initial electron is launched at $x=a / 2$, and the simulation is run 42 times, corresponding to 42 equidistant phases of the RF field. The mean value of the final population of electrons after 20 impacts of the ee against the walls is calculated using all the 42 simulations. Also, the secondary

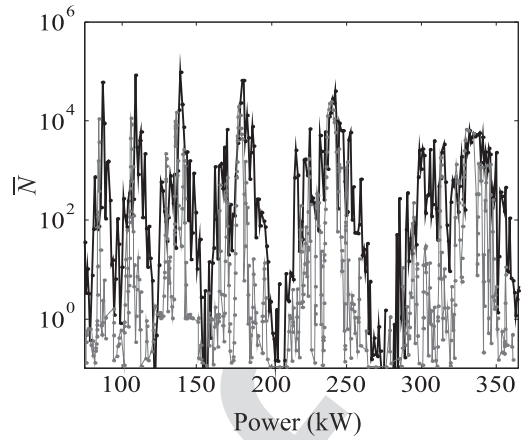

Fig. 2. Comparison with [14] of the mean value of $N$ over all launch phases in a rectangular waveguide $(a=43.1 \mathrm{~cm}$ and $b=10.2 \mathrm{~cm})$ driven at $f=500 \mathrm{MHz}$ with a maximum of 20 impacts from a single initial launch location on the midline of the empty rectangular waveguide.

electrons generated after every collision are launched with an energy of $2 \mathrm{eV}$ normal to the impacting surface. The maximum simulation lifetime of each ee is $t_{\max }=1000 \mathrm{RF}$ cycles, and the simulation is stopped if the impact energy is lower than $0.1 \mathrm{eV}$ or if the accumulated population of electrons is under $10^{-3}$. To model the same simulation conditions, our CAD tool has been adapted accordingly. In Fig. 2, the results of the mean population of electrons, $\bar{N}$, computed with our code (black lines) are compared with the curves presented in [14] (gray lines). In this figure, we can see some high-risk multipactor power regions. Both curves show a good agreement in the shape and location of these multipactor windows.

Once the model has been validated for an empty waveguide, next we analyze the multipactor effect in a partially dielectric-loaded rectangular waveguide. The selected waveguide configuration for the multipactor analysis is a nonstandard silver-plated rectangular waveguide of width $a=$ $19.05 \mathrm{~mm}$ and heigth $b=0.4 \mathrm{~mm}$, in which a thin dielectric layer has been placed over the bottom surface of the waveguide. A realistic dielectric material has been chosen as teflon (DuPont Teflon fluorinated ethylene propylene Fluoroplastic Film Type), which is a dielectric film commonly used in space applications, of thickness $h=0.025 \mathrm{~mm}$ and $\epsilon_{r}=2.1$; thus $d=b-h=0.375 \mathrm{~mm}$. Standard values for the SEY parameters of silver [31] are given in Table I, and SEY parameters of teflon have been measured at the ESA-VSC High Power Space Materials Laboratory (Valencia, Spain) [32]. First, a study of the susceptibility chart of this waveguide has been performed. Since it is a partially dielectric-loaded rectangular waveguide, the factor $f \times d$ is plotted in the horizontal axis of the susceptibility chart. In the vertical axis, it is plotted an effective voltage, $V_{\text {eff }}$, which has been calculated numerically as the line integral of the $E_{y}$ component of the electric field (evaluated at the center of the waveguide $x=a / 2$ ) from $y_{1}=0$ to $y_{2}=d$. To obtain this susceptibility chart for each $V_{\text {eff }}$ and $f \times d$ pair, the simulation is run 72 times, corresponding to 72 equidistant initial phases of the RF field separated $5^{\circ}$. In each run, an initial single ee is launched at $x=a / 2$ and $z=0$ and at a random position $y_{0}$ in the $y$-axis between $y=0$ and $y=d$. The initial electron is launched with a departure kinetic energy given by the probability density function shown in (17) and following 


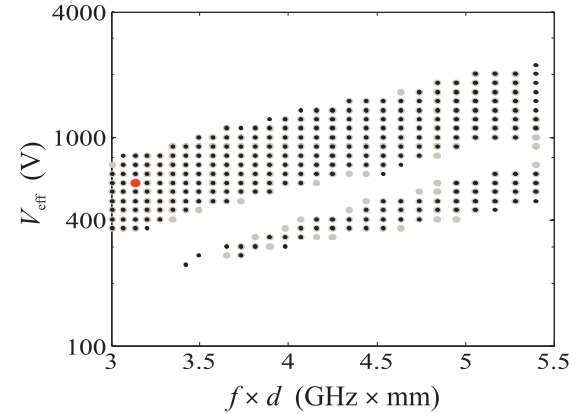

Fig. 3. Comparison of the susceptibility chart of a rectangular waveguide partially filled with teflon (black points) with that of its equivalent empty waveguide (gray points). Red: operating point corresponding to $f \times d=$ $3.13 \mathrm{GHz} \cdot \mathrm{mm}$ and $V_{\text {eff }}=608 \mathrm{~V}$.

a cosine distribution of the polar angle. Each simulation was stopped after $100 \mathrm{RF}$ cycles. In the empty waveguide, the arithmetic mean of the final population of electrons after $100 \mathrm{RF}$ cycles is calculated using all the 72 simulations. If this mean value is greater than 1 , then the multipactor discharge is assumed to have occurred. However, in a partially dielectric-loaded waveguide, it has been shown in previous works [8]-[10] that the emission or absorption of electrons by the dielectric surface gives rise to an increasing dc field in the waveguide, which eventually turns off the discharge. Thus, in this case, a minimum mean value of the magnitude of $E_{\mathrm{dc}}$ field in the waveguide after $100 \mathrm{RF}$ cycles is used as the criterion to assume that a multipactor discharge has occurred at a given operating point.

Fig. 3 shows the computed susceptibility chart of the rectangular waveguide partially filled with teflon (black points). The lowest $f \times d$ value is above the cutoff frequency of the fundamental mode in this waveguide. In this figure, the susceptibility chart of the equivalent empty waveguide with the same vertical air gap is also represented with gray points for comparison. It can be checked that both the empty and the partially dielectric-loaded waveguide with the same vertical air gap show similar multipactor susceptibility charts, given that the SEY properties of silver and teflon are similar. This susceptibility chart is not generally applicable to any rectangular waveguide with an air gap $d$, given that the electromagnetic field distribution depends on the geometry and dimensions of the dielectric layer with respect to the waveguide dimensions, and also on its relative permittivity.

From the results shown earlier, and with the purpose of having a better understanding of the dynamics of the electron inside the partially dielectric-loaded waveguide, a point within the multipactor region has been chosen (highlighted in red in Fig. 3), corresponding to $V_{\text {eff }}=608 \mathrm{~V}$ and $f \times d=$ $3.13 \mathrm{GHz} \cdot \mathrm{mm}$. In this case, the evolution of the multipactor discharge in the partially dielectric-loaded waveguide under study has been analyzed as a function of the time normalized to the RF period. For this simulation, the electron is launched with an initial phase of the RF field $\varphi_{0}=0^{\circ}$. Simulations assuming different initial phases have been performed, and similar results were obtained. The obtained simulation is shown in Fig. 4, where it is plotted the $y$-coordinate followed by the ee within the waveguide as a function of the normalized

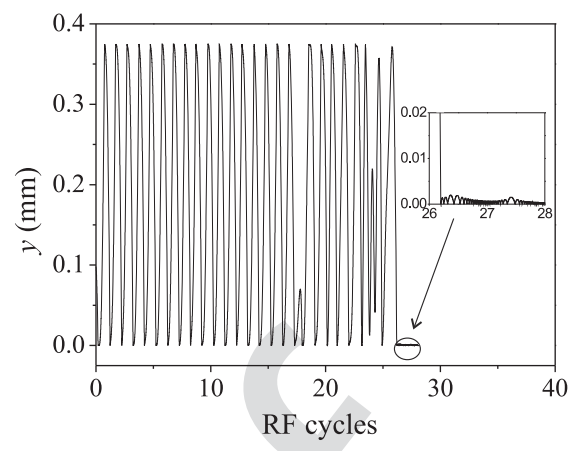

Fig. 4. Trajectory ( $y$-coordinate) of the ee in the air gap of the rectangular waveguide partially filled with teflon as a function of the RF cycle.

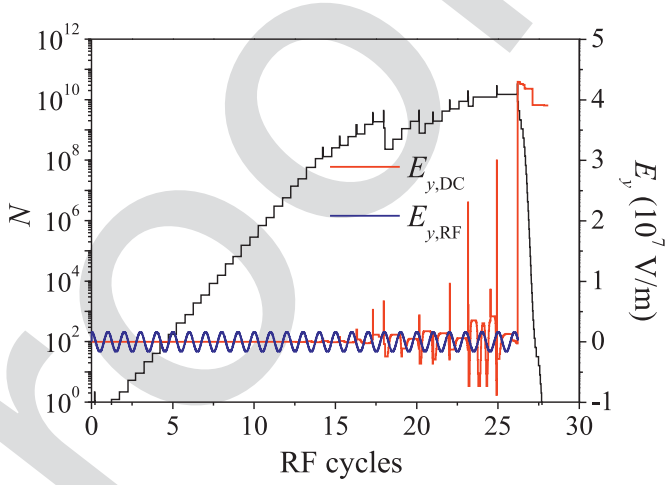

Fig. 5. Black line: time evolution of the total number of electrons $N$. Blue line: $E_{y, \mathrm{RF}}$. Red line: $E_{y, \mathrm{dc}}$ at the electron position.

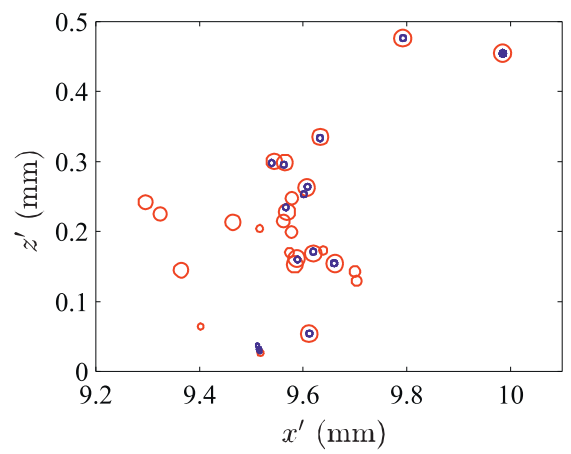

Fig. 6. Distribution of normalized charges $\bar{Q}_{i}=Q_{i} / e$ appearing on the dielectric surface.

time. In the selected multipactor regime, which is inside the multipactor region, the electron initially collides with the top metallic and bottom dielectric surface consecutively in what seems to be a first-order multipactor process during the first $17 \mathrm{RF}$ cycles, remaining in the vicinity of $x=$ $a / 2$ and $z=0$-given that the electron has nearly no acceleration in such directions. As shown in Fig. 5, in the first cycles, the total number of electrons $N$ (black solid line) follows an exponential growth. This progressive growing of $N$ makes that the number of charges appearing on the dielectric surface increases, the number which is proportional to the emitted or absorbed electrons in each impact, as seen in Fig. 6 (positive charges are represented with red circles, while negative charges are represented with blue circles; the circles' size is proportional to the charge magnitude in log scale). Such charges on the dielectric interface give rise to the 


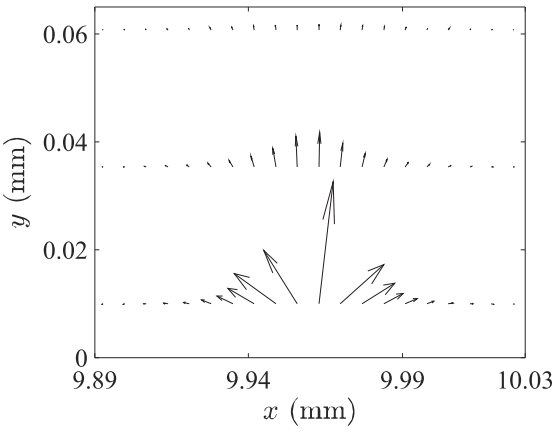

Fig. 7. Transverse distribution of the dc electric field in the proximity of the main charge point in the waveguide air region at RF cycle 26.2 , at $z=$ $0.45 \mathrm{~mm}$.

appearance of an electrostatic field in the empty gap during the time between impacts. Once the population of electrons reaches a significant number $\left(N \approx 10^{9}\right.$ in the conditions under study), the $y$-component of the dc field, $E_{y, \mathrm{dc}}$ [which has been plotted in Fig. 5 with red line at the positions $(x, y, z)$ where the effective electron is located in the displayed instants in this figure] becomes comparable to $E_{y, \mathrm{RF}}$, and the effective electron is unable to keep up with its previous multipactor synchronization. From this moment on, the dc field makes that, in some impacts, the electrons collide with the top metallic or bottom dielectric surface much sooner or later than the instants when the RF electric field changes its sign, which implies low impact energy collisions so that electrons are absorbed in such impacts. In collisions at the dielectric surface, the absorption or emission of electrons yield the appearance of growing charges on the dielectric layer, contributing to a higher dc field acting on the waveguide. The distribution of this high dc field in the proximity of the main charge point in the waveguide air region is shown in Fig. 7 at RF cycle 26.2 in the plane $z=0.45 \mathrm{~mm}$ (corresponding to the $z$ position of the electron at this instant). The action of this field may result in the appearance of a single-surface multipactor regime in the dielectric surface [see the $y$ position of the electron in Fig. 4 (inset) from RF cycle 26.2], with successive low impact energy collisions, which eventually leads to the turning off of the discharge itself (as can be appreciated in Fig. 5 from RF cycle 26.2 on). From this instant, the dc field distribution in the waveguide remains nearly constant, given that $N$ drops very quickly. The final value of the $y$-component of the dc field accounts for the balance between the emitted and absorbed electrons by the dielectric surface in the whole process. Then, although the final population of electrons after RF cycle 100 is 0 , the remaining high dc field in the waveguide indicates that a multipactor discharge has taken place in the waveguide in this simulation. It is worth mentioning that the observed turning OFF of the discharge observed in the last stages of the multipactor evolution in this waveguide has been speeded up due to the use of the EEM, although this does not change qualitatively the dynamics of the discharge under these conditions.

\section{CONClusion}

A study of the multipactor effect in a partially dielectricloaded rectangular waveguide has been carried out. In this paper, we have considered the RF electromagnetic fields (obtained with a very efficient vectorial modal method) as well as the dc field caused by the appearance of a charge distribution in the dielectric layer. The solution of the electrostatic problem has required the use of different numerical integration techniques and interpolation methods. The electron trajectory has been numerically solved by using a Velocity Verlet algorithm, providing sufficient accuracy and good efficiency. As a first example, the multipactor prediction results of an empty rectangular waveguide have been obtained for validation purposes. Second, the susceptibility chart of a partially dielectric-loaded rectangular waveguide has been computed, and the time evolution of a discharge in this waveguide has been studied and discussed. The performed simulations reveal that multipactor discharges in this type of dielectric-loaded waveguides turn OFF by themselves due to the electrostatic field associated with the dielectric surface charges that evolve with the multipactor process.

\section{REFERENCES}

[1] J. R. M. Vaughan, "Multipactor," IEEE Trans. Electron Devices, vol. ED-35, no. 7, pp. 1172-1180, Jul. 1988, doi: 10.1109/16.3387.

[2] V. E. Semenov et al., "Simulations of multipactor thresholds in shielded microstrip lines," J. Phys. D, Appl. Phys., vol. 42, no. 20 pp. 205204-1-205204-7, Sep. 2009, doi: 10.1109/TMAG.2017.2658671

[3] A. M. Pérez et al., "Prediction of multipactor breakdown thresholds in coaxial transmission lines for traveling, standing, and mixed waves," IEEE Trans. Plasma Sci., vol. 37, no. 10, pp. 2031-2040, Oct. 2009 , doi: 10.1109/TPS.2009.2028428.

[4] R. Kishek, Y. Y. Lau, L. K. Ang, A. Valfells, and R. M. Gilgenbach, "Multipactor discharge on metals and dielectrics: Historical review and recent theories," Phys. Plasmas, vol. 5, no. 5, pp. 2120-2126, May 1998, doi: $10.1063 / 1.872883$

[5] L.-K. Ang, Y. Y. Lau, R. A. Kishek, and R. M. Gilgenbach, "Power deposited on a dielectric by multipactor," IEEE Trans. Plasma Sci., vol. 26, no. 3, pp. 290-295, Jun. 1998, doi: 10.1109/27.700756.

[6] A. Valfells, L. K. Ang, Y. Y. Lau, and R. M. Gilgenbach, "Effects of an external magnetic field, and of oblique radio-frequency electric fields on multipactor discharge on a dielectric," Phys. Plasmas, vol. 7, no. 2 pp. 750-757, Feb. 2000, doi: 10.1063/1.873861.

[7] J. G. Power et al., "Observation of multipactor in an alumina-based dielectric-loaded accelerating structure," Phys. Rev. Lett., vol. 92 no. 16, pp. 164801-1-164801-4, Apr. 2004, doi: 10.1103/PhysRevLett.92.164801.

[8] G. Torregrosa, A. Coves, C. P. Vicente, A. M. Pérez, B. Gimeno, an V. E. Boria, "Time evolution of an electron discharge in a parallel-plate dielectric-loaded waveguide," IEEE Electron Device Lett., vol. 27, no. 7, pp. 619-621, Jul. 2006, doi: 10.1109/LED.2006.877284.

[9] A. Coves, G. Torregrosa-Penalva, C. Vicente, B. Gimeno, and V. E. Boria, "Multipactor discharges in parallel-plate dielectricloaded waveguides including space-charge effects," IEEE Trans. Electron Devices, vol. 55, no. 9, pp. 2505-2511, Sep. 2008 , doi: 10.1109/TED.2008.927945.

[10] G. Torregrosa, Á. Coves, B. G. Martinez, I. Montero, C. Vicente, and V. E. Boria, "Multipactor susceptibility charts of a parallel-plate dielectric-loaded waveguide," IEEE Trans. Electron Devices, vol. 57, no. 5, pp. 1160-1166, May 2010, doi: 10.1109/TED.2010.2043182.

[11] D. González-Iglesias et al., "Multipactor in a coaxial line under the presence of an axial DC magnetic field," IEEE Electron Device Lett. vol. 33, no. 5, pp. 727-729, May 2012, doi: 10.1109/LED.2012.2186952.

[12] D. González-Iglesias et al. " Multipactor mitigation in coaxial lines by means of permanent magnets," IEEE Trans. Electron Devices, vol. 61 no. 12, pp. 4224-4231, Dec. 2014, doi: 10.1109/TED.2014.2361172.

[13] V. E. Semenov, E. I. Rakova, D. Anderson, M. Lisak, and J. Puech, "Multipactor in rectangular waveguides," Phys. Plasmas, vol. 14, no. 3, pp. 033501-1-033501-8, Mar. 2007, doi: 10.1063/1.2480678.

[14] E. Chojnacki, "Simulations of a multipactor-inhibited waveguide geometry," Phys. Rev. Special Topics Accel. Beams, vol. 3, no. 3, p. 032001 , Mar. 2000, doi: 10.1103/PhysRevSTAB.3.032001. 


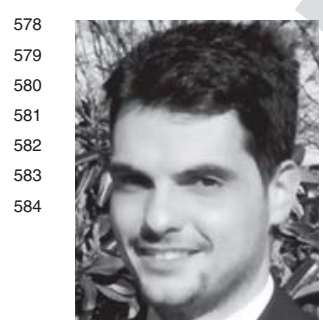
Pearson, 2008. Apr. 2000.
[15] E. Somersalo, D. Proch, P. Ylä-Oijala, and J. Sarvas, "Computational methods for analyzing electron multipacting in RF structures," Part. Accel., vol. 95, pp. 107-141, Jan. 1998.

16] M. Buyanova, V. E. Semenov, D. Anderson, M. Lisak, and J. Puech, "Influence of secondary emission yield on the saturation properties of multipactor discharges between two parallel metal plates," Phys. Plasmas, vol. 17, p. 043504, Mar. 2010, doi: 10.1063/1.3374430.

[17] C. J. Lingwood, G. Burt, A. C. Dexter, J. D. A. Smith, P. Goudket, and P. H. Stoltz, "Phase space analysis of multipactor saturation in rectangular waveguide," Phys. Plasmas, vol. 19, p. 032106, Feb. 2012, doi: 10.1063/1.3692060.

[18] R. F. Harrington, Time-Harmonic Electromagnetic Fields. Hoboken, NJ, USA: Wiley, 2001.

[19] C. A. Balanis, Advanced Engineering Electromagnetics, 2nd ed. Hoboken, NJ, USA: Wiley, 2012.

[20] D. J. Griffiths, Introduction to Electrodynamics, 3rd ed. London, U.K.

[21] R. E. Collin, Field Theory of Guided Waves, 2nd ed. Piscataway, NJ, USA: IEEE Press, 1990.

[22] A. Berenguer, A. Coves, F. Mesa, E. Bronchalo, B. Gimeno, and V. Boria, "Calculation of the electrostatic field in a dielectric-loaded waveguide due to an arbitrary charge distribution on the dielectric layer," in Proc. PIERS, Shanghai, China, Aug. 2016, pp. 3251-3255, doi: 10.1109/PIERS.2016.7735274.

[23] A. Berenguer, A. Coves, E. Bronchalo, B. Gimeno, and V. E. Boria, "Analysis of multipactor effect in parallel-plate and rectangular waveguides," in Proc. PIERS, Prague, Czech Republic, Jul. 2015, pp. 1564-1568, doi: 10.1063/1.2480678.

$24]$ J. R. M. Vaughan, "A new formula for secondary emission yield," IEEE Trans. Electron Devices, vol. 36, no. 9, pp. 1963-1967, Sep. 1989, doi: $10.1109 / 16.34278$

[25] C. Vicente et al., "Multipactor breakdown prediction in rectangular waveguide based components," in IEEE MTT-S Int. Microw. Symp. Dig., Long Beach, CA, USA, Jun. 2005, pp. 1055-1058, doi: 10.1109/MWSYM.2005.1516852.

[26] R. Seviour, "The role of elastic and inelastic electron reflection in multipactor discharges," IEEE Trans. Electron Devices, vol. 52, no. 8 , pp. 1927-1930, Aug. 2005, doi: 10.1109/TED.2005.851854.

[27] R. Cimino et al., "Can low-energy electrons affect high-energy physics accelerators?" Phys. Rev. Lett., vol. 93, no. 1, p. 014801, 2004, doi: 10.1103/PhysRevLett.93.014801.

[28] E. Bronchalo et al., "Secondary electron emission of Pt: Experimental study and comparison with models in the multipactor energy range," IEEE Trans. Electron Devices, vol. 63, no. 8, pp. 3270-3277, Aug. 2016, doi: 10.1109/TED.2016.2580199.

[29] J. J. Scholtz, D. Dijkkamp, and R. W. A. Schmitz, "Secondary electron emission properties," Philips J. Res., vol. 50, nos. 3-4, pp. 375-389, 1996, doi: 10.1016/S0165-5817(97)84681-5.

[30] R. L. Geng and H. S. Padamsee, "Exploring multipacting characteristics of a rectangular waveguide," in Proc. Part. Accel. Conf., New York, NY, USA, Mar./Apr. 1999, pp. 429-431, doi: 10.1109/PAC.1999.795724.

[31] Multipacting Design and Test, document ESTEC ECSS-20-01,

[32] SEY Measurements of PTFE. Accessed: Feb. 2014. [Online]. Available: www.val-space.com

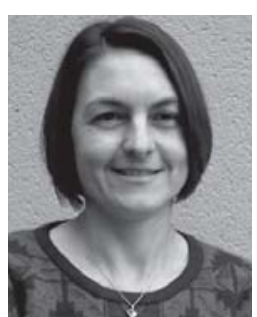

Angela Coves (S'04-M'05) received the Licenciado and $\mathrm{Ph} . \mathrm{D}$. degrees in physics from the Universidad de Valencia, Valencia, Spain, in 1999 and 2004, respectively.

She is currently an Associate Professor with the Miguel Hernandez University of Elche, Alicante, Spain. Her current research interests include microwave passive components and RF breakdown high-power effects.

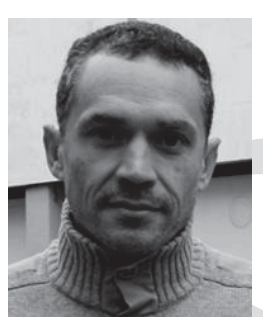

Francisco Mesa (M'93-SM'11-F'14) received the Licenciado and Ph.D. degrees in physics from the Universidad de Seville, Seville, Spain, in 1989 and 1991, respectively.

He is currently a Professor with the Departamento de Física Aplicada 1, Universidad de Seville. Hi current research interests include electromagnetic propagation/radiation in planar structures.

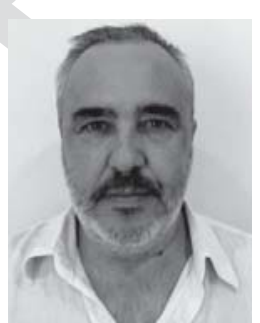

Enrique Bronchalo received the Physics degree from the Universidad Complutense de Madrid, Madrid, Spain, in 1986, and the Ph.D. degree on Physics from the Universidad de Alcalá, Alcalá, Spain, in 1996.

$\mathrm{He}$ is currently an Associate Professor with the Miguel Hernandez University of Elche, Alicante, Spain. His current research interests include on passive microwave devices and multipactor processes in waveguides.

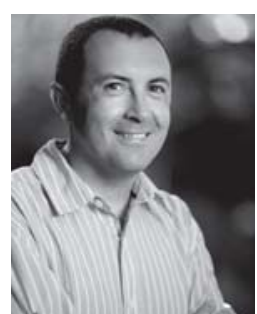

Benito Gimeno (M'01) received the Licenciado and Ph.D. degrees in physics from the University of Valencia, Valencia, Spain, in 1987 and 1992 respectively.

$\mathrm{He}$ is currently a Professor with the University of Valencia. His current research interests include microwave passive components and RF breakdown high-power effects. 


\title{
Analysis of Multipactor Effect in a Partially Dielectric-Loaded Rectangular Waveguide
}

\author{
Andrés Berenguer, Ángela Coves ${ }^{\circledR}$, Member, IEEE, Francisco Mesa $^{\circledR}$, Fellow, IEEE, Enrique Bronchalo ${ }^{\circledR}$, \\ and Benito Gimeno ${ }^{\circledR}$, Member, IEEE
}

\begin{abstract}
This paper presents a study of the multipactor effect in a partially dielectric-loaded rectangular waveguide. To obtain the simulations presented in this paper, a detailed analysis of the dynamics of the electron inside this waveguide has been performed, taking into account the radio frequency electromagnetic fields propagating in the waveguide and the dc electric field that appears because of the charging of the dielectric layer. This electrostatic field is obtained by computing the electric potential produced by an arbitrary charge distribution on the dielectric layer in a dielectric-loaded waveguide. The electron trajectory is then found by numerically solving the equations of motion. The results obtained show that multipactor discharges do turn off by themselves under certain circumstances when they occur in such dielectric-loaded waveguide.
\end{abstract}

Index Terms-Multipactor, secondary emission, waveguide.

\section{INTRODUCTION}

$\mathbf{M}$ ULTIPACTOR is a high-power resonant electron discharge frequently observed in the microwave and millimeter-wave subsystems operating under vacuum conditions [1] present in a wide range of different scenarios, such as passive components of satellite communication payloads, traveling-wave, tubes or particle accelerators. In an ultrahigh vacuum environment, the free electrons inside a microwave device are accelerated by the radio frequency (RF) electromagnetic fields, impacting against its metallic walls. If the electron impact energy is high enough, one or more secondary electrons might be released from the surface. When some resonance conditions are satisfied, the secondary electrons get synchronized with the RF fields, and the electron population inside the device grows exponentially leading to a multipactor discharge. This multipactor discharge has some negative effects that degrade the device performance: increase

Manuscript received May 9, 2018; revised October 3, 2018; accepted November 4, 2018. This work was supported in part by the Agencia Estatal de Investigación and in part by the Unión Europea through the Fondo Europeo de Desarrollo Regional-Una Manera de Hacer Europa under Project TEC2016-75934-C4-2-R and Project TEC2017-84724-P. The review of this paper was arranged by Senior Editor S. J. Gitomer. (Corresponding author: Angela Coves.)

A. Berenguer, A. Coves, and E. Bronchalo are with the Department of Communication Engineering, Miguel Hernandez University of Elche, 03202 Alicante, Spain (e-mail: angela.coves@umh.es).

F. Mesa is with the Microwaves Group, Department of Applied Physics 1,

Escuela Técnica Superior de Ingenieria Informatica, Universidad de Seville, 41004 Seville, Spain.

B. Gimeno is with the Department of Applied Physics and Electromagnetism, Institute of Materials Science, Universidad de Valencia, 46010 València, Spain.

Color versions of one or more of the figures in this paper are available online at http://ieeexplore.ieee.org.

Digital Object Identifier 10.1109/TPS.2018.2880652 of signal noise and reflected power, heating of the device walls, outgassing, detuning of resonant cavities, and even the partial or total destruction of the component.

Multipactor research lines are aimed to study and characterize the phenomenon to predict the conditions for its appearance [2], [3]. Some RF devices, such as filters, multiplexers, and RF satellite payloads, include dielectric materials commonly employed as resonators and supporting elements. In [4], it is presented a review of multipactor discharge on metals and dielectric windows that takes into account the surface materials, and the effects of space charge and cavity loading. The multipactor effect including the presence of dielectric materials in single-surface multipactor regime has been widely investigated in the context of particle accelerators; for instance, in ceramic RF windows [5], [6] and in aluminabased dielectric-loaded accelerating structures [7]. In contrast, very few contributions can be found about multipactor breakdown on dielectrics in the scenario of RF systems for space applications [8]-[10], and mostly under the parallelplate waveguide approximation. In [11] and [12], the effective electron model (EEM) has been successfully used for simulations of multipaction experiments in coaxial transmission lines considering the presence of external magnetic static fields, demonstrating the validity of this method in complex scenarios. The multipactor inside an empty rectangular waveguide has also been studied in [13] and [14], where the conventional resonance theory gives correct predictions for the multipactor threshold if the height of the waveguide is very small and first-order resonance multipactor dominates. When the waveguide height exceeds a certain critical value, which depends on the waveguide width, an accurate prediction of the multipactor threshold requires considering the RF fields inside the waveguide without approximations. Therefore, there is a need to accurately predict the electron discharge on devices involving partially dielectric-loaded rectangular waveguides, which are of more practical interest for satellite technology. The main aim of this investigation is to extend the results of previous works [8]-[10], where an EEM was successfully applied to study the multipactor in a parallel-plate dielectricloaded waveguide, to the analysis of multipactor effect in a partially dielectric-loaded rectangular waveguide.

In Section II, the theoretical model employed for the simulations is discussed. In Section III, the multipactor prediction results of an empty rectangular waveguide are analyzed and compared with results from the technical literature for validation purposes. Then, the susceptibility chart of a partially 


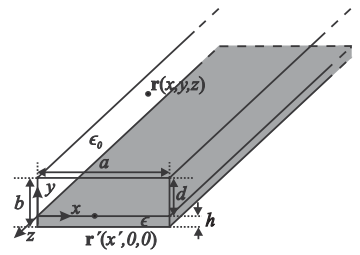

Fig. 1. Geometry and dimensions of the problem under investigation.

dielectric-loaded rectangular waveguide is obtained with the developed model, and the time evolution of a discharge in this waveguide is studied and discussed. Finally, Section IV outlines the main conclusions of this paper.

\section{THEORY}

\section{A. Computation of RF and DC Fields in a Partially Dielectric-Loaded Waveguide}

Fig. 1 shows the scheme of a partially dielectric-loaded rectangular waveguide of width $a$ and height $b$, and whose dielectric material has relative permittivity $\epsilon_{r}$. In the problem under study, the dielectric slab of thickness $h$ and width $a$ is placed over the bottom waveguide wall, being $d$ the empty waveguide height where the effective electron travels (see Fig. 1). The RF electromagnetic field is assumed to propagate along the positive direction of the $z$-axis. For the sake of simplicity, the waveguide is supposed to be infinite along the $z$ direction, and a time-harmonic dependence of the type $e^{j \omega t}$ is implicitly assumed, with $f=\omega / 2 \pi$ being the frequency and $t$ the time measured in the laboratory reference system. To analyze the multipactor evolution in this waveguide, a multipactor simulation code based on the Monte Carlo method has been developed. The software code, similar to the one described in [8] and [9], employs the single EEM [15]. This assumption avoids the consideration of space-charge effects, what is a strong simplification. The space-charge effects are often neglected in the analysis of the first stages of the multipactor discharge [13], [14], but they are doubtless important at high electron populations when the discharge is fully developed. Simulation results of some published works [16], [17] indicate an important role of space-charge in the evolution of the multipactor process to a saturation stage. In this paper, however, we are mainly interested in studying the influence of dielectric charging in the multipactor process. The inclusion of space-charge effects, although providing a more realistic description of the global process, would increase the computational burden very much, as the dc field due to dielectric charging has to be evaluated in every effective electron position. In addition, the interpretation of simulation results would become difficult, as dielectric charging and space charge can both lead to a repulsion of the freshly emitted secondary electrons back to the surface.

The effective electron at $\mathbf{r}=(x, y, z)$ can move in the air region of height $d$ of the rectangular waveguide. The electromagnetic fields $\mathbf{E}_{\mathrm{RF}}$ and $\mathbf{H}_{\mathrm{RF}}$ acting on the effective electron correspond to the modes of the partially dielectricloaded rectangular waveguide (Fig. 1), which are hybrid modes of $\mathrm{TM}^{y}$ and $\mathrm{TE}^{y}$ kinds [18]. We have restricted our study to the monomode regime, where only the fundamental mode, $\mathrm{TM}_{10}^{y}$, propagates in the waveguide. The instantaneous field vectors interacting with the effective electron are given by

$$
\begin{aligned}
& \mathbf{E}_{\mathrm{RF}}(x, y, z, t)=E_{0} \Re\left(\mathbf{e}(x, y) e^{j\left(\omega t-\beta z+\varphi_{0}\right)}\right) \\
& \mathbf{H}_{\mathrm{RF}}(x, y, z, t)=H_{0} \Re\left(\mathbf{h}(x, y) e^{j\left(\omega t-\beta z+\varphi_{0}\right)}\right)
\end{aligned}
$$

where $\varphi_{0}$ is the initial phase and $E_{0}, H_{0}$ are the constants related to the transmitted power in the waveguide. The modal fields $\mathbf{e}(x, y)$ and $\mathbf{h}(x, y)$ and the propagation constant $\beta$ of the $\mathrm{TM}_{10}^{y}$ mode can be found in [18] and [19]. These expressions can be directly extended if higher order modes must be taken into account (i.e., in waveguide discontinuities) by using the mode-matching technique.

The key to understanding the mechanism of a multipactor discharge is to study the behavior of the electrons within the waveguide, which are accelerated by the aforementioned electromagnetic fields $\mathbf{E}_{\mathrm{RF}}$ and $\mathbf{H}_{\mathrm{RF}}$. In this way, sooner or later, these fields will make an electron impact with any surface of the rectangular waveguide, which can result in the emission or absorption of secondary electrons. If the impacts occur on the dielectric surface, unlike the case of impacts on the metallic walls, the secondary electrons emitted by the dielectric give rise to positive charges at the impact positions on the dielectric surface, while the electrons absorbed in the dielectric layer will generate negative charges in it. These charges, which are located on the dielectric surface at positions $\mathbf{r}^{\prime}=\left(x^{\prime}, 0, z^{\prime}\right)$, give rise to an electrostatic field $\mathbf{E}_{\mathrm{dc}}$, which has to be added to the RF fields to obtain accurately the trajectory of the electrons inside the waveguide. In order to determine the electrostatic field, $\mathbf{E}_{\mathrm{dc}}(x, y, z)=-\nabla \phi(x, y, z)$, generated by the charges on the dielectric, the potential $\phi(x, y, z)$ inside the waveguide has to be first calculated. Using the superposition, the potential in the waveguide due to the set of charges $Q_{i}$ on the dielectric surface can be obtained by adding the individual contribution of each charge

$$
\phi(x, y, z)=\sum_{i} G\left(x-x_{i}^{\prime}, y,\left|z-z_{i}^{\prime}\right|\right) Q_{i}\left(x_{i}^{\prime}, 0, z_{i}^{\prime}\right)
$$

where $G(x, y, z)$ is the electrostatic potential due to a unit point charge, that is, Green's function for this problem.

The above-mentioned Green's function, $G(x, y, z)$, is the solution to the following Laplace's equation [20], [21]:

$$
\nabla \cdot\left[\epsilon_{r}(y) \nabla G(x, y, z)\right]=-\frac{1}{\epsilon_{0}} \delta\left(x-x^{\prime}\right) \delta(y) \delta(z)
$$

where $\epsilon_{0}$ is the free-space dielectric permittivity and the position of the unit charge is taken at $\left(x^{\prime}, 0,0\right)$ for convenience. Both the geometric characteristics and the linear nature of the problem under consideration makes that the Dirac delta functions can be expressed as [21]

$$
\begin{aligned}
\delta\left(x-x^{\prime}\right) & =\frac{2}{a} \sum_{n=1}^{\infty} \sin \left(k_{x n} x\right) \sin \left(k_{x n} x^{\prime}\right) \\
\delta(z) & =\frac{1}{2 \pi} \int_{-\infty}^{\infty} e^{-j k_{z} z} d k_{z}
\end{aligned}
$$

where $k_{x n}=n \pi / a$ and $k_{z}$ is the spectral Fourier variable along the longitudinal direction $z$. The above-mentioned expressions 
come from the fact that the eigenfunctions of the differential operator are sinusoidal functions along $x$-axis and complex exponential functions along the $z$-axis, respectively. This is equivalent to apply the discrete sine transform along the $x$-axis and the integral transform along the $z$-axis, namely,

$$
\begin{aligned}
G & =\frac{1}{\pi a} \int_{-\infty}^{\infty} d k_{z} e^{-j k_{z} z} \sum_{n=1}^{\infty} \sin \left(k_{x n} x\right) \sin \left(k_{x n} x^{\prime}\right) \widetilde{G} \\
\widetilde{G} & =\int_{-\infty}^{\infty} d z e^{j k_{z} z} \sum_{n=1}^{\infty} \sin \left(k_{x n} x\right) \sin \left(k_{x n} x^{\prime}\right) G
\end{aligned}
$$

where $G=G\left(x, x^{\prime}, y, z\right)$ and $\widetilde{G}=\widetilde{G}\left(k_{x n}, k_{z} ; y\right)$.

According to the above-mentioned considerations, (3) can be expressed as the following ordinary differential equation for the spectral Green's function $\widetilde{G}$ :

$$
\begin{aligned}
\left\{\frac{d}{d y} \epsilon_{r}(y) \frac{d}{d y}-k_{t}^{2}\right\} \widetilde{G} & =-\frac{\delta(y)}{\epsilon_{0}} \\
\widetilde{G}(y=-h) & =0 \\
\widetilde{G}(y=d) & =0
\end{aligned}
$$

where $k_{t}^{2}=k_{x n}^{2}+k_{z}^{2}$. Solving (8), the following expression for $\widetilde{G}$ is obtained in the air region $y \geq 0$ :

$$
\widetilde{G}\left(k_{x n}, k_{z} ; y\right)=\frac{\sinh \left[k_{t}(d-y)\right]}{\epsilon_{0} k_{t}\left[\epsilon_{r} \operatorname{coth}\left(k_{t} h\right)+\operatorname{coth}\left(k_{t} d\right)\right] \sinh \left(k_{t} d\right)} .
$$

Green's function in the spatial domain, $G$, is achieved by replacing (9) into (6) to give

$$
\begin{aligned}
& G\left(x, x^{\prime}, y, z\right) \\
& =\frac{2}{\epsilon_{0} \pi a} \sum_{n=1}^{\infty} \sin \left(k_{x n} x\right) \sin \left(k_{x n} x^{\prime}\right) \\
& \quad \times \int_{0}^{\infty} \frac{\sinh \left[k_{t}(d-y)\right] \cos \left(k_{z} z\right)}{k_{t}\left[\epsilon_{r} \operatorname{coth}\left(k_{t} h\right)+\operatorname{coth}\left(k_{t} d\right)\right] \sinh \left(k_{t} d\right)} d k_{z} .
\end{aligned}
$$

In (10), if the point charge is placed at $z^{\prime} \neq 0, z$ must be replaced by $\left(z-z^{\prime}\right)$. Here, it is worth noting that very efficient numerical summation and integration techniques have to be employed to compute Green's function with sufficient accuracy and tolerable CPU times [22].

Once Green's function has been calculated, the $\mathbf{E}_{\mathrm{dc}}$ field is obtained by numerical differentiation of (2) by means of the central difference technique.

\section{B. Multipactor Evolution in the Partially Dielectric-Loaded Waveguide}

Once the RF and dc fields are known at any instant $t$, the electron dynamics inside the waveguide can be computed, which is governed by the Lorentz force and related to its linear momentum

$$
\mathbf{F}_{L}=q(\mathbf{E}+\mathbf{v} \times \mathbf{B})=\frac{d \mathbf{p}}{d t}
$$

where $q=-e$ is the electron charge, $\mathbf{E}$ and $\mathbf{B}=\mu_{0} \mathbf{H}$ are the total electric and magnetic fields (both RF and dc contributions) interacting with the electron, $\mu_{0}$ is the freespace magnetic permeability, and $\mathbf{v}$ is the velocity vector of the electron. The linear relativistic momentum is defined as

$$
\mathbf{p}=m_{0} \gamma \mathbf{v}
$$

where $m_{0}$ is the electron rest mass, $\gamma=1 / \sqrt{1-(v / c)^{2}}$ the Lorentz factor, $v$ is the magnitude of the velocity vector, and $c=1 / \sqrt{\mu_{0} \epsilon_{0}}$ the speed of light in vacuum. Although the relativistic correction in this equation can be discarded for the typical power ranges of most space waveguide devices, it must be considered when high velocities are reached $(v / c \geq 0.1)$, as in high-power multipactor simulations. Expanding (11) the following differential equation is obtained:

$$
-\mathbf{E}-\mathbf{v} \times \mathbf{B}=M \gamma \mathbf{a}+\frac{M}{c^{2}} \gamma^{3}(\mathbf{v} \cdot \mathbf{a}) \mathbf{v}
$$

where $\mathbf{a}$ is the acceleration vector and $M=m_{0} / e$. The differential equation to be solved becomes

$$
\ddot{\mathbf{r}}=\frac{-\dot{\mathbf{r}} \times \mathbf{B}-\mathbf{E}+\dot{\mathbf{r}} \cdot(\dot{\mathbf{r}} \cdot \mathbf{E}) / c^{2}}{M \gamma} .
$$

The electron trajectory is found by numerically solving the above-mentioned equations of motion. For that purpose, a Velocity Verlet algorithm [23] has been used, which assures sufficient accuracy and good efficiency provided the time step is small enough. Regarding this last point, in order to improve the accuracy and efficiency of the simulation, the following adaptive time step has been applied in the proximity of the waveguide walls, depending on the electron position:

$$
\Delta t=\frac{\Delta t_{0}}{1+\xi\left(\frac{x-a / 2}{a / 2}\right)^{2}+\xi\left(\frac{y-d / 2}{d / 2}\right)^{2}}
$$

where $\Delta t_{0}$ is the initial reference time step, $\xi$ is a constant value (in this case, a value of 4.0 has been chosen), and $x$ and $y$ are the coordinates of the electron position.

As mentioned above, the computed electrons trajectories may lead to an eventual impact with a surface. Each collision can result in the emission or absorption of secondary electrons. A relevant growth in the electron density can develop if the electrons hit the walls with the appropriate energy and at suitable instants. The number of electrons emitted or absorbed after each impact is determined by the value of the Secondary Electron Yield (SEY) parameter $\delta(\delta>1$ if secondary electrons are emitted, and $\delta<1$ if they are absorbed). The SEY is modeled by a modification of Vaughan's model [24] that includes the effect of reflected electrons for low impact energies of primary electrons, which has to be accounted for to obtain accurate results [25], [26] in agreement with experimental data obtained in [27], [28]. The SEY properties for surface materials can be defined by the following parameters: the primary electron impact kinetic energies which yield $\delta=1$, $W_{1}$, and $W_{2}$; the impact energy $W_{\max }$ necessary for a primary electron to yield $\delta=\delta_{\max }$, which is the maximum value of the SEY function; and the value of the primary electron impact energy $W_{0}(\delta=0)$ that limits the region of elastic collisions.

When a multipactor discharge evolves in the partially dielectric-loaded waveguide under study, the dc field distribution has to be updated after each electron impacts on the 
TABLE I

SECONDARY ELECTRON EMISSION YIELd PROPERTIES of DifFERENT MATERIALs [31], [32]

\begin{tabular}{|c|c|c|c|c|c|}
\hline Material & $\mathbf{W}_{\max }(\mathbf{e V})$ & $\mathbf{W}_{\mathbf{1}}(\mathbf{e V})$ & $\mathbf{W}_{\mathbf{2}}(\mathbf{e V})$ & $\mathbf{W}_{\mathbf{0}}(\mathbf{e V})$ & $\delta_{\max }$ \\
\hline Niobium & 200.0 & 33 & 1500 & - & 1.6 \\
\hline Silver & 165.0 & 30 & 5000 & 15.99 & 2.22 \\
\hline Teflon & 271.7 & 27 & 5000 & 6.81 & 2.47 \\
\hline
\end{tabular}

dielectric surface. However, tracking the evolution of all the electrons involved in the multipactor discharge would suppose a big computational cost. Thus, we have made use of an EEM, but considering both the spread in secondary emission energy and the angle of the secondary electrons after each impact on the waveguide walls. This assumption has proved to account properly for the charging of the dielectric material, given that the discharging time for dielectrics is much higher than the typical time for a multipactor discharge. Thus, in the EEM assumed in this paper, after the effective electron impacts at time $t$ with any surface, $N_{i}(t)$ is modified according to the $\delta$ value provided by the SEY function as follows:

$$
N_{i}(t+\Delta t)=\delta N_{i}(t)
$$

where $N_{i}(t)$ represents the population of the ee inside the waveguide at the instant $t$, and $\Delta t$ is the time step used in the simulations.

The secondary electron departure kinetic energy $E_{s}$ after each electron impact is assumed to fit the following probability density function [29]:

$$
\frac{d p\left(E_{S}\right)}{d E_{S}}=C \exp \left[-\frac{\ln ^{2}\left(E_{S} / E_{m}\right)}{2 \tau^{2}}\right]
$$

where $C$ is a normalization constant, the parameter $\tau$ (typical values $0.7-0.8$ ) determines the width of the distribution and $E_{m}$ (typical values 3-4 eV) is the energy of the maximum of the spectrum. Finally, the secondary electrons after inelastic impacts are emitted following a cosine distribution of the polar angle.

\section{NUMERICAL RESULTS AND DISCUSSION}

An in-house simulation computer-aided design (CAD) tool based on the Monte Carlo method described in Section II has been developed to analyze the multipactor effect in partially dielectric-loaded rectangular waveguides. The first problem analyzed consists of an empty rectangular waveguide previously studied in [14], whose multipactor prediction results have been used for validation purposes. The rectangular waveguide has dimensions $a=43.2 \mathrm{~cm}$ and $b=10.2 \mathrm{~cm}$, and is excited by a time-harmonic signal at $f=500 \mathrm{MHz}$. The material of the waveguide walls is niobium, whose SEY properties are given in Table I and can be expressed with the simple model proposed in [30]. In the algorithm of the simulator used in [14], for each RF power considered in the waveguide, the initial electron is launched at $x=a / 2$, and the simulation is run 42 times, corresponding to 42 equidistant phases of the RF field. The mean value of the final population of electrons after 20 impacts of the ee against the walls is calculated using all the 42 simulations. Also, the secondary

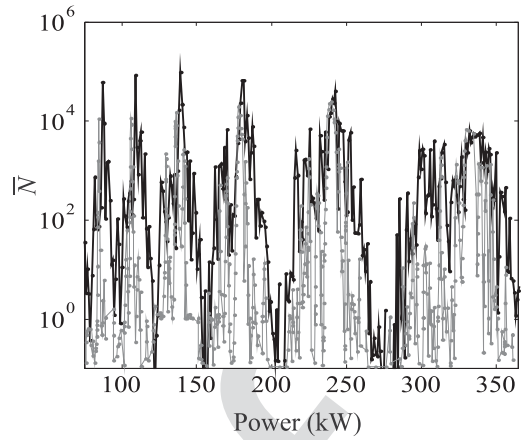

Fig. 2. Comparison with [14] of the mean value of $N$ over all launch phases in a rectangular waveguide $(a=43.1 \mathrm{~cm}$ and $b=10.2 \mathrm{~cm})$ driven at $f=500 \mathrm{MHz}$ with a maximum of 20 impacts from a single initial launch location on the midline of the empty rectangular waveguide.

electrons generated after every collision are launched with an energy of $2 \mathrm{eV}$ normal to the impacting surface. The maximum simulation lifetime of each ee is $t_{\max }=1000 \mathrm{RF}$ cycles, and the simulation is stopped if the impact energy is lower than $0.1 \mathrm{eV}$ or if the accumulated population of electrons is under $10^{-3}$. To model the same simulation conditions, our CAD tool has been adapted accordingly. In Fig. 2, the results of the mean population of electrons, $\bar{N}$, computed with our code (black lines) are compared with the curves presented in [14] (gray lines). In this figure, we can see some high-risk multipactor power regions. Both curves show a good agreement in the shape and location of these multipactor windows.

Once the model has been validated for an empty waveguide, next we analyze the multipactor effect in a partially dielectric-loaded rectangular waveguide. The selected waveguide configuration for the multipactor analysis is a nonstandard silver-plated rectangular waveguide of width $a=$ $19.05 \mathrm{~mm}$ and heigth $b=0.4 \mathrm{~mm}$, in which a thin dielectric layer has been placed over the bottom surface of the waveguide. A realistic dielectric material has been chosen as teflon (DuPont Teflon fluorinated ethylene propylene Fluoroplastic Film Type), which is a dielectric film commonly used in space applications, of thickness $h=0.025 \mathrm{~mm}$ and $\epsilon_{r}=2.1$; thus $d=b-h=0.375 \mathrm{~mm}$. Standard values for the SEY parameters of silver [31] are given in Table I, and SEY parameters of teflon have been measured at the ESA-VSC High Power Space Materials Laboratory (Valencia, Spain) [32]. First, a study of the susceptibility chart of this waveguide has been performed. Since it is a partially dielectric-loaded rectangular waveguide, the factor $f \times d$ is plotted in the horizontal axis of the susceptibility chart. In the vertical axis, it is plotted an effective voltage, $V_{\text {eff }}$, which has been calculated numerically as the line integral of the $E_{y}$ component of the electric field (evaluated at the center of the waveguide $x=a / 2$ ) from $y_{1}=0$ to $y_{2}=d$. To obtain this susceptibility chart for each $V_{\text {eff }}$ and $f \times d$ pair, the simulation is run 72 times, corresponding to 72 equidistant initial phases of the RF field separated $5^{\circ}$. In each run, an initial single ee is launched at $x=a / 2$ and $z=0$ and at a random position $y_{0}$ in the $y$-axis between $y=0$ and $y=d$. The initial electron is launched with a departure kinetic energy given by the probability density function shown in (17) and following 


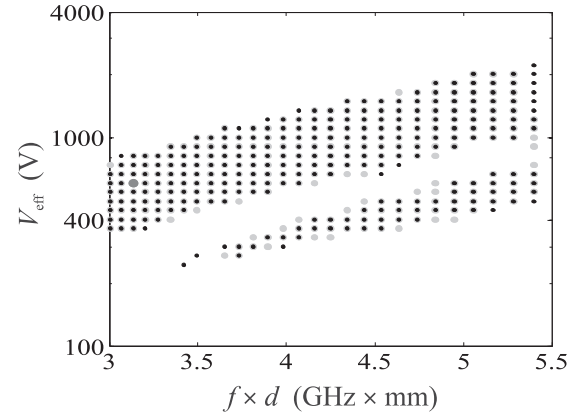

Fig. 3. Comparison of the susceptibility chart of a rectangular waveguide partially filled with teflon (black points) with that of its equivalent empty waveguide (gray points). Red: operating point corresponding to $f \times d=$ $3.13 \mathrm{GHz} \cdot \mathrm{mm}$ and $V_{\text {eff }}=608 \mathrm{~V}$.

a cosine distribution of the polar angle. Each simulation was stopped after $100 \mathrm{RF}$ cycles. In the empty waveguide, the arithmetic mean of the final population of electrons after $100 \mathrm{RF}$ cycles is calculated using all the 72 simulations. If this mean value is greater than 1, then the multipactor discharge is assumed to have occurred. However, in a partially dielectric-loaded waveguide, it has been shown in previous works [8]-[10] that the emission or absorption of electrons by the dielectric surface gives rise to an increasing dc field in the waveguide, which eventually turns off the discharge. Thus, in this case, a minimum mean value of the magnitude of $E_{\mathrm{dc}}$ field in the waveguide after $100 \mathrm{RF}$ cycles is used as the criterion to assume that a multipactor discharge has occurred at a given operating point.

Fig. 3 shows the computed susceptibility chart of the rectangular waveguide partially filled with teflon (black points). The lowest $f \times d$ value is above the cutoff frequency of the fundamental mode in this waveguide. In this figure, the susceptibility chart of the equivalent empty waveguide with the same vertical air gap is also represented with gray points for comparison. It can be checked that both the empty and the partially dielectric-loaded waveguide with the same vertical air gap show similar multipactor susceptibility charts, given that the SEY properties of silver and teflon are similar. This susceptibility chart is not generally applicable to any rectangular waveguide with an air gap $d$, given that the electromagnetic field distribution depends on the geometry and dimensions of the dielectric layer with respect to the waveguide dimensions, and also on its relative permittivity.

From the results shown earlier, and with the purpose of having a better understanding of the dynamics of the electron inside the partially dielectric-loaded waveguide, a point within the multipactor region has been chosen (highlighted in red in Fig. 3), corresponding to $V_{\text {eff }}=608 \mathrm{~V}$ and $f \times d=$ $3.13 \mathrm{GHz} \cdot \mathrm{mm}$. In this case, the evolution of the multipactor discharge in the partially dielectric-loaded waveguide under study has been analyzed as a function of the time normalized to the RF period. For this simulation, the electron is launched with an initial phase of the RF field $\varphi_{0}=0^{\circ}$. Simulations assuming different initial phases have been performed, and similar results were obtained. The obtained simulation is shown in Fig. 4, where it is plotted the $y$-coordinate followed by the ee within the waveguide as a function of the normalized

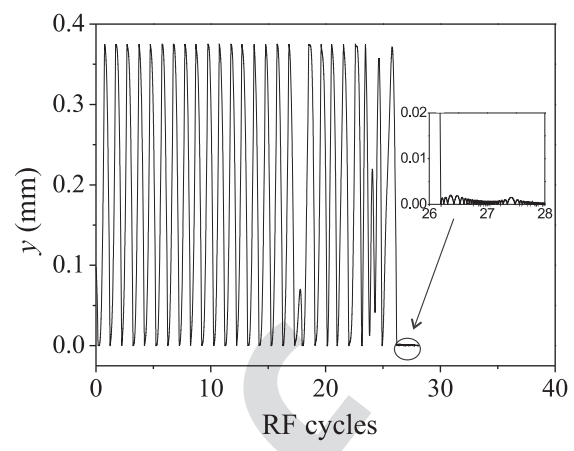

Fig. 4. Trajectory ( $y$-coordinate) of the ee in the air gap of the rectangular waveguide partially filled with teflon as a function of the RF cycle.

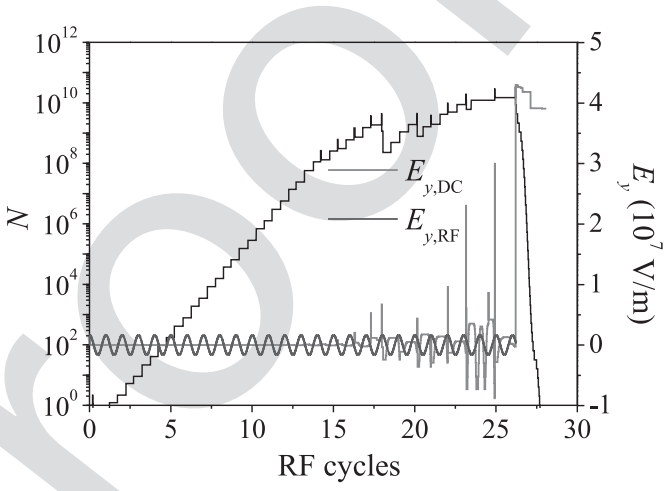

Fig. 5. Black line: time evolution of the total number of electrons $N$. Blue line: $E_{y}, \mathrm{RF}$. Red line: $E_{y}$,dc at the electron position.

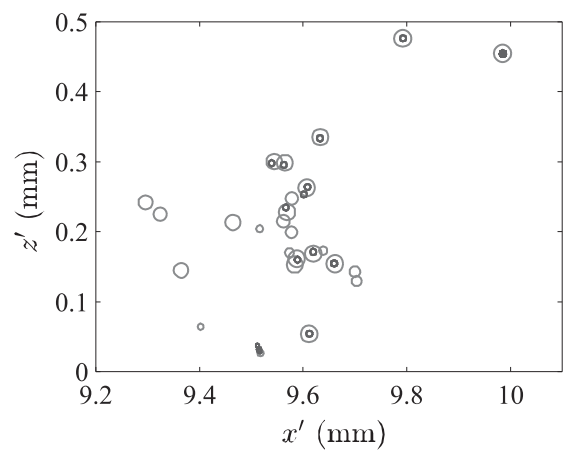

Fig. 6. Distribution of normalized charges $\bar{Q}_{i}=Q_{i} / e$ appearing on the dielectric surface.

time. In the selected multipactor regime, which is inside the multipactor region, the electron initially collides with the top metallic and bottom dielectric surface consecutively in what seems to be a first-order multipactor process during the first $17 \mathrm{RF}$ cycles, remaining in the vicinity of $x=$ $a / 2$ and $z=0$-given that the electron has nearly no acceleration in such directions. As shown in Fig. 5, in the first cycles, the total number of electrons $N$ (black solid line) follows an exponential growth. This progressive growing of $N$ makes that the number of charges appearing on the dielectric surface increases, the number which is proportional to the emitted or absorbed electrons in each impact, as seen in Fig. 6 (positive charges are represented with red circles, while negative charges are represented with blue circles; the circles' size is proportional to the charge magnitude in log scale). Such charges on the dielectric interface give rise to the 


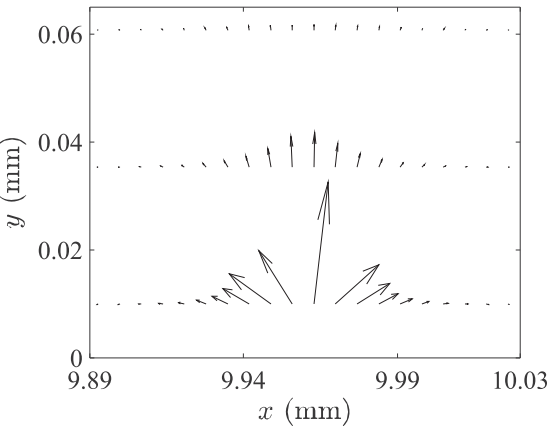

Fig. 7. Transverse distribution of the dc electric field in the proximity of the main charge point in the waveguide air region at RF cycle 26.2 , at $z=$ $0.45 \mathrm{~mm}$.

appearance of an electrostatic field in the empty gap during the time between impacts. Once the population of electrons reaches a significant number $\left(N \approx 10^{9}\right.$ in the conditions under study), the $y$-component of the dc field, $E_{y, \text { dc }}$ [which has been plotted in Fig. 5 with red line at the positions $(x, y, z)$ where the effective electron is located in the displayed instants in this figure] becomes comparable to $E_{y, \mathrm{RF}}$, and the effective electron is unable to keep up with its previous multipactor synchronization. From this moment on, the dc field makes that, in some impacts, the electrons collide with the top metallic or bottom dielectric surface much sooner or later than the instants when the RF electric field changes its sign, which implies low impact energy collisions so that electrons are absorbed in such impacts. In collisions at the dielectric surface, the absorption or emission of electrons yield the appearance of growing charges on the dielectric layer, contributing to a higher dc field acting on the waveguide. The distribution of this high dc field in the proximity of the main charge point in the waveguide air region is shown in Fig. 7 at RF cycle 26.2 in the plane $z=0.45 \mathrm{~mm}$ (corresponding to the $z$ position of the electron at this instant). The action of this field may result in the appearance of a single-surface multipactor regime in the dielectric surface [see the $y$ position of the electron in Fig. 4 (inset) from RF cycle 26.2], with successive low impact energy collisions, which eventually leads to the turning off of the discharge itself (as can be appreciated in Fig. 5 from RF cycle 26.2 on). From this instant, the dc field distribution in the waveguide remains nearly constant, given that $N$ drops very quickly. The final value of the $y$-component of the dc field accounts for the balance between the emitted and absorbed electrons by the dielectric surface in the whole process. Then, although the final population of electrons after RF cycle 100 is 0 , the remaining high dc field in the waveguide indicates that a multipactor discharge has taken place in the waveguide in this simulation. It is worth mentioning that the observed turning OFF of the discharge observed in the last stages of the multipactor evolution in this waveguide has been speeded up due to the use of the EEM, although this does not change qualitatively the dynamics of the discharge under these conditions.

\section{CONClusion}

A study of the multipactor effect in a partially dielectricloaded rectangular waveguide has been carried out. In this paper, we have considered the RF electromagnetic fields (obtained with a very efficient vectorial modal method) as well as the dc field caused by the appearance of a charge distribution in the dielectric layer. The solution of the electrostatic problem has required the use of different numerical integration techniques and interpolation methods. The electron trajectory has been numerically solved by using a Velocity Verlet algorithm, providing sufficient accuracy and good efficiency. As a first example, the multipactor prediction results of an empty rectangular waveguide have been obtained for validation purposes. Second, the susceptibility chart of a partially dielectric-loaded rectangular waveguide has been computed, and the time evolution of a discharge in this waveguide has been studied and discussed. The performed simulations reveal that multipactor discharges in this type of dielectric-loaded waveguides turn OFF by themselves due to the electrostatic field associated with the dielectric surface charges that evolve with the multipactor process.

\section{REFERENCES}

[1] J. R. M. Vaughan, "Multipactor," IEEE Trans. Electron Devices, vol. ED-35, no. 7, pp. 1172-1180, Jul. 1988, doi: 10.1109/16.3387.

[2] V. E. Semenov et al., "Simulations of multipactor thresholds in shielded microstrip lines," J. Phys. D, Appl. Phys., vol. 42, no. 20 pp. 205204-1-205204-7, Sep. 2009, doi: 10.1109/TMAG.2017.2658671

[3] A. M. Pérez et al., "Prediction of multipactor breakdown thresholds in coaxial transmission lines for traveling, standing, and mixed waves," IEEE Trans. Plasma Sci., vol. 37, no. 10, pp. 2031-2040, Oct. 2009, doi: 10.1109/TPS.2009.2028428.

[4] R. Kishek, Y. Y. Lau, L. K. Ang, A. Valfells, and R. M. Gilgenbach, "Multipactor discharge on metals and dielectrics: Historical review and recent theories," Phys. Plasmas, vol. 5, no. 5, pp. 2120-2126, May 1998, doi: 10.1063/1.872883.

[5] L.-K. Ang, Y. Y. Lau, R. A. Kishek, and R. M. Gilgenbach, "Power deposited on a dielectric by multipactor," IEEE Trans. Plasma Sci. vol. 26, no. 3, pp. 290-295, Jun. 1998, doi: 10.1109/27.700756.

[6] A. Valfells, L. K. Ang, Y. Y. Lau, and R. M. Gilgenbach, "Effects of an external magnetic field, and of oblique radio-frequency electric fields on multipactor discharge on a dielectric," Phys. Plasmas, vol. 7, no. 2, pp. 750-757, Feb. 2000, doi: 10.1063/1.873861.

[7] J. G. Power et al., "Observation of multipactor in an alumina-based dielectric-loaded accelerating structure," Phys. Rev. Lett., vol. 92 , no. 16, pp. 164801-1-164801-4, Apr. 2004, doi: 10.1103/PhysRevLett.92.164801

[8] G. Torregrosa, A. Coves, C. P. Vicente, A. M. Pérez, B. Gimeno, an V. E. Boria, "Time evolution of an electron discharge in a parallel-plate dielectric-loaded waveguide," IEEE Electron Device Lett., vol. 27, no. 7 pp. 619-621, Jul. 2006, doi: 10.1109/LED.2006.877284.

[9] A. Coves, G. Torregrosa-Penalva, C. Vicente, B. Gimeno, and V. E. Boria, "Multipactor discharges in parallel-plate dielectricloaded waveguides including space-charge effects," IEEE Trans. Electron Devices, vol. 55, no. 9, pp. 2505-2511, Sep. 2008 , doi: 10.1109/TED.2008.927945.

[10] G. Torregrosa, Á. Coves, B. G. Martinez, I. Montero, C. Vicente, and V. E. Boria, "Multipactor susceptibility charts of a parallel-plate dielectric-loaded waveguide," IEEE Trans. Electron Devices, vol. 57 no. 5, pp. 1160-1166, May 2010, doi: 10.1109/TED.2010.2043182.

[11] D. González-Iglesias et al., "Multipactor in a coaxial line under the presence of an axial DC magnetic field," IEEE Electron Device Lett. vol. 33, no. 5, pp. 727-729, May 2012, doi: 10.1109/LED.2012.2186952.

[12] D. González-Iglesias et al., "Multipactor mitigation in coaxial lines by means of permanent magnets," IEEE Trans. Electron Devices, vol. 61 no. 12, pp. 4224-4231, Dec. 2014, doi: 10.1109/TED.2014.2361172.

[13] V. E. Semenov, E. I. Rakova, D. Anderson, M. Lisak, and J. Puech, "Multipactor in rectangular waveguides," Phys. Plasmas, vol. 14, no. 3, pp. 033501-1-033501-8, Mar. 2007, doi: 10.1063/1.2480678.

[14] E. Chojnacki, "Simulations of a multipactor-inhibited waveguide geometry," Phys. Rev. Special Topics Accel. Beams, vol. 3, no. 3, p. 032001 , Mar. 2000, doi: 10.1103/PhysRevSTAB.3.032001. 


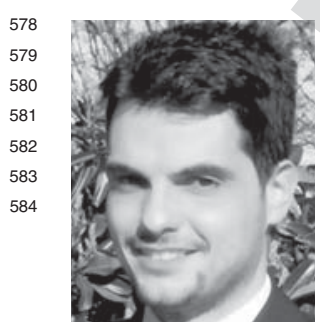
Pearson, 2008. Apr. 2000.
[15] E. Somersalo, D. Proch, P. Ylä-Oijala, and J. Sarvas, "Computational methods for analyzing electron multipacting in RF structures," Part. Accel., vol. 95, pp. 107-141, Jan. 1998.

[16] M. Buyanova, V. E. Semenov, D. Anderson, M. Lisak, and J. Puech, "Influence of secondary emission yield on the saturation properties of multipactor discharges between two parallel metal plates," Phys. Plasmas, vol. 17, p. 043504, Mar. 2010, doi: 10.1063/1.3374430.

[17] C. J. Lingwood, G. Burt, A. C. Dexter, J. D. A. Smith, P. Goudket, and P. H. Stoltz, "Phase space analysis of multipactor saturation in rectangular waveguide,” Phys. Plasmas, vol. 19, p. 032106, Feb. 2012, doi: $10.1063 / 1.3692060$.

[18] R. F. Harrington, Time-Harmonic Electromagnetic Fields. Hoboken, NJ, USA: Wiley, 2001.

[19] C. A. Balanis, Advanced Engineering Electromagnetics, 2nd ed. Hoboken, NJ, USA: Wiley, 2012.

[20] D. J. Griffiths, Introduction to Electrodynamics, 3rd ed. London, U.K.

[21] R. E. Collin, Field Theory of Guided Waves, 2nd ed. Piscataway, NJ, USA: IEEE Press, 1990.

[22] A. Berenguer, A. Coves, F. Mesa, E. Bronchalo, B. Gimeno, and V. Boria, "Calculation of the electrostatic field in a dielectric-loaded waveguide due to an arbitrary charge distribution on the dielectric layer," in Proc. PIERS, Shanghai, China, Aug. 2016, pp. 3251-3255, doi: 10.1109/PIERS.2016.7735274.

[23] A. Berenguer, A. Coves, E. Bronchalo, B. Gimeno, and V. E. Boria, "Analysis of multipactor effect in parallel-plate and rectangular waveguides," in Proc. PIERS, Prague, Czech Republic, Jul. 2015, pp. 1564-1568, doi: 10.1063/1.2480678.

$24]$ J. R. M. Vaughan, "A new formula for secondary emission yield," IEEE Trans. Electron Devices, vol. 36, no. 9, pp. 1963-1967, Sep. 1989, doi: $10.1109 / 16.34278$

[25] C. Vicente et al., "Multipactor breakdown prediction in rectangular waveguide based components," in IEEE MTT-S Int. Microw. Symp. Dig., Long Beach, CA, USA, Jun. 2005, pp. 1055-1058, doi: 10.1109/MWSYM.2005.1516852

[26] R. Seviour, "The role of elastic and inelastic electron reflection in multipactor discharges," IEEE Trans. Electron Devices, vol. 52, no. 8, pp. 1927-1930, Aug. 2005, doi: 10.1109/TED.2005.851854.

[27] R. Cimino et al., "Can low-energy electrons affect high-energy physics accelerators?" Phys. Rev. Lett., vol. 93, no. 1, p. 014801, 2004, doi: 10.1103/PhysRevLett.93.014801.

[28] E. Bronchalo et al., "Secondary electron emission of Pt: Experimental study and comparison with models in the multipactor energy range," IEEE Trans. Electron Devices, vol. 63, no. 8, pp. 3270-3277, Aug. 2016, doi: 10.1109/TED.2016.2580199.

[29] J. J. Scholtz, D. Dijkkamp, and R. W. A. Schmitz, "Secondary electron emission properties," Philips J. Res., vol. 50, nos. 3-4, pp. 375-389, 1996, doi: 10.1016/S0165-5817(97)84681-5.

[30] R. L. Geng and H. S. Padamsee, "Exploring multipacting characteristics of a rectangular waveguide," in Proc. Part. Accel. Conf., New York, NY, USA, Mar./Apr. 1999, pp. 429-431, doi: 10.1109/PAC.1999.795724.

[31] Multipacting Design and Test, document ESTEC ECSS-20-01,

32] SEY Measurements of PTFE. Accessed: Feb. 2014. [Online]. Available: www.val-space.com

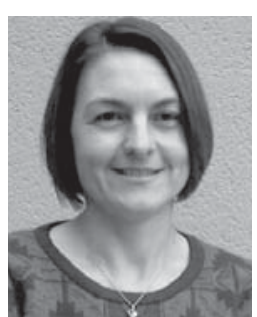

Angela Coves (S'04-M'05) received the Licenciado and $\mathrm{Ph} . \mathrm{D}$. degrees in physics from the Universidad de Valencia, Valencia, Spain, in 1999 and 2004, respectively.

She is currently an Associate Professor with the Miguel Hernandez University of Elche, Alicante, Spain. Her current research interests include microwave passive components and RF breakdown high-power effects.

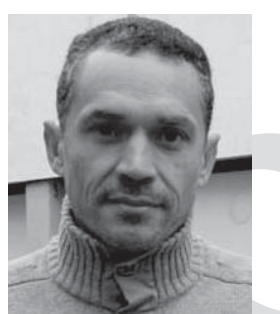

Francisco Mesa (M'93-SM'11-F'14) received the Licenciado and Ph.D. degrees in physics from the Universidad de Seville, Seville, Spain, in 1989 and 1991, respectively.

He is currently a Professor with the Departamento de Física Aplicada 1, Universidad de Seville. His current research interests include electromagnetic propagation/radiation in planar structures.

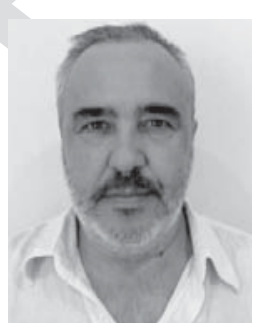

Enrique Bronchalo received the Physics degree from the Universidad Complutense de Madrid Madrid, Spain, in 1986, and the Ph.D. degree on Physics from the Universidad de Alcalá, Alcalá, Spain, in 1996.

$\mathrm{He}$ is currently an Associate Professor with the Miguel Hernandez University of Elche, Alicante, Spain. His current research interests include on passive microwave devices and multipactor processes in waveguides.

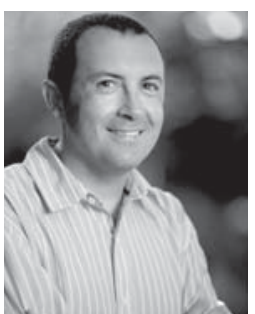

Benito Gimeno (M'01) received the Licenciado and Ph.D. degrees in physics from the University of Valencia, Valencia, Spain, in 1987 and 1992 respectively.

$\mathrm{He}$ is currently a Professor with the University of Valencia. His current research interests include microwave passive components and RF breakdown high-power effects. 